\title{
Molecular dynamics analysis of conformational change of paramyxovirus $F$ protein during the initial steps of membrane fusion
}

\author{
Fernando Martín-García a,b , Jesús Ignacio Mendieta-Moreno ${ }^{\mathrm{a}, \mathrm{b}}$, Jesús Mendieta ${ }^{\mathrm{a}, \mathrm{b}}$, \\ Paulino Gómez-Puertas ${ }^{\mathrm{a}, *}$ \\ a Centro de Biología Molecular “Severo Ochoa" (CSIC/UAM), C/ Nicolás Cabrera, 1, Cantoblanco, 28049 Madrid, Spain \\ ${ }^{\mathrm{b}}$ Biomol-Informatics SL, Parque Científico de Madrid, C/ Faraday, 7, Cantoblanco, 28049 Madrid, Spain
}

\section{A R T I C L E I N F O}

\section{Article history:}

Received 1 February 2012

Available online 28 February 2012

\section{Keywords:}

Molecular dynamics

Fusion protein

Conformational change

Mechanical force

\begin{abstract}
A B S T R A C T
The fusion of paramyxovirus to the cell membrane is mediated by fusion protein (F protein) present in the virus envelope, which undergoes a dramatic conformational change during the process. Unlike hemagglutinin in orthomyxovirus, this change is not mediated by an alteration of environmental $\mathrm{pH}$, and its cause remains unknown. Steered molecular dynamics analysis leads us to suggest that the conformational modification is mediated only by stretching mechanical forces once the transmembrane fusion peptide of the protein is anchored to the cell membrane. Such elongating forces will generate major secondary structure rearrangement in the heptad repeat A region of the F protein; from $\beta$-sheet conformation to an elongated coil and then spontaneously to an $\alpha$-helix. In addition, it is proposed that the heptad repeat A region adopts a final three-helix coiled coil and that this structure appears after the formation of individual helices in each monomer.
\end{abstract}

(C) 2012 Elsevier Inc. All rights reserved.

\section{Introduction}

The Paramyxoviridae family is composed of enveloped, negative-stranded RNA viruses, many of which are human pathogens (e.g., the measles virus, human respiratory syncytial virus (RSV), Hendra and Nipah viruses and human metapneumovirus [1]). Their entry into target cells is mediated by two glycoproteins located on the viral membrane: the attachment $(G)$ protein, that binds to heparin sulfate, and the fusion $(F)$ protein $[2,3]$. The presence of both proteins and their interaction is usually necessary to drive the membrane fusion event [4-6]; however, both human and bovine $\mathrm{RSV}$, as well as human metapneumovirus (MPV), are exceptions: their membranes can fuse in the absence of the viral $G$ protein [7-10].

The F protein belongs to the class I viral fusion proteins, whose most important representative is from the influenza virus hemagglutinin (HA). Each monomer is initially synthesized as the F0 precursor and subsequently cleaved into the active F1 + F2 heterodimer by a trypsine-like protease. This cleavage releases a transmembrane fusion peptide ( $F$ peptide) with a length of 25-48 residues, located in the $\mathrm{N}$-terminal end of $\mathrm{F} 1$ [11]. After binding

Abbreviations: F protein, fusion protein; MD, molecular dynamics; HRA, heptad repeat $A$; HRB, heptad repeat $B$.

* Corresponding author. Fax: +34 911964420.

E-mail address: pagomez@cbm.uam.es (P. Gómez-Puertas). to the host cell membrane, the trimeric F1 + F2 complex experiences a major multi-step structural change, mainly in the heptad repeat $A$ (HRA) and heptad repeat $B$ (HRB) domains, which results in a completely different post-fusion structure [12]. The change is irreversible; blocked by the energy barrier of the membrane fusion [13].

In the case of the Paramyxoviridae family, crystal structures have recently been published for both pre-fusion (parainfluenza virus 5; PDB ID: 2B9B [14]) and post-fusion (human parainfluenza virus 3; PDB ID: 1ZTM [15]) conformations of two viruses, and they illustrate this dramatic change. It has been suggested that other members of the family undergo the same structural modification, based on their sequence similarities. A multiple sequence alignment of domain III (DIII) of the F protein from some viruses in this family to illustrate their similarity is included as Supplementary material (Supp. Fig. S1). The HRA region of the protein undergoes major change in its secondary structure, and thus in its 3D structure. From an initial folded state composed of two beta strands in anti-parallel conformation, followed by a short $\alpha$-helix segment (Supp. Fig. S1), the post-fusion arrangement of the HRA segment has a completely refolded structure. This post-fusion structure incorporates the residues that were formerly part of the 2beta + 1alpha region into a newly formed, completely extended all-alpha structure (Supp. Fig. S1) that interacts with the helix formed by the other two monomers, thus generating a three-helix coiled coil $[15,16]$. Although the rest of the structure of DIII is also altered, 

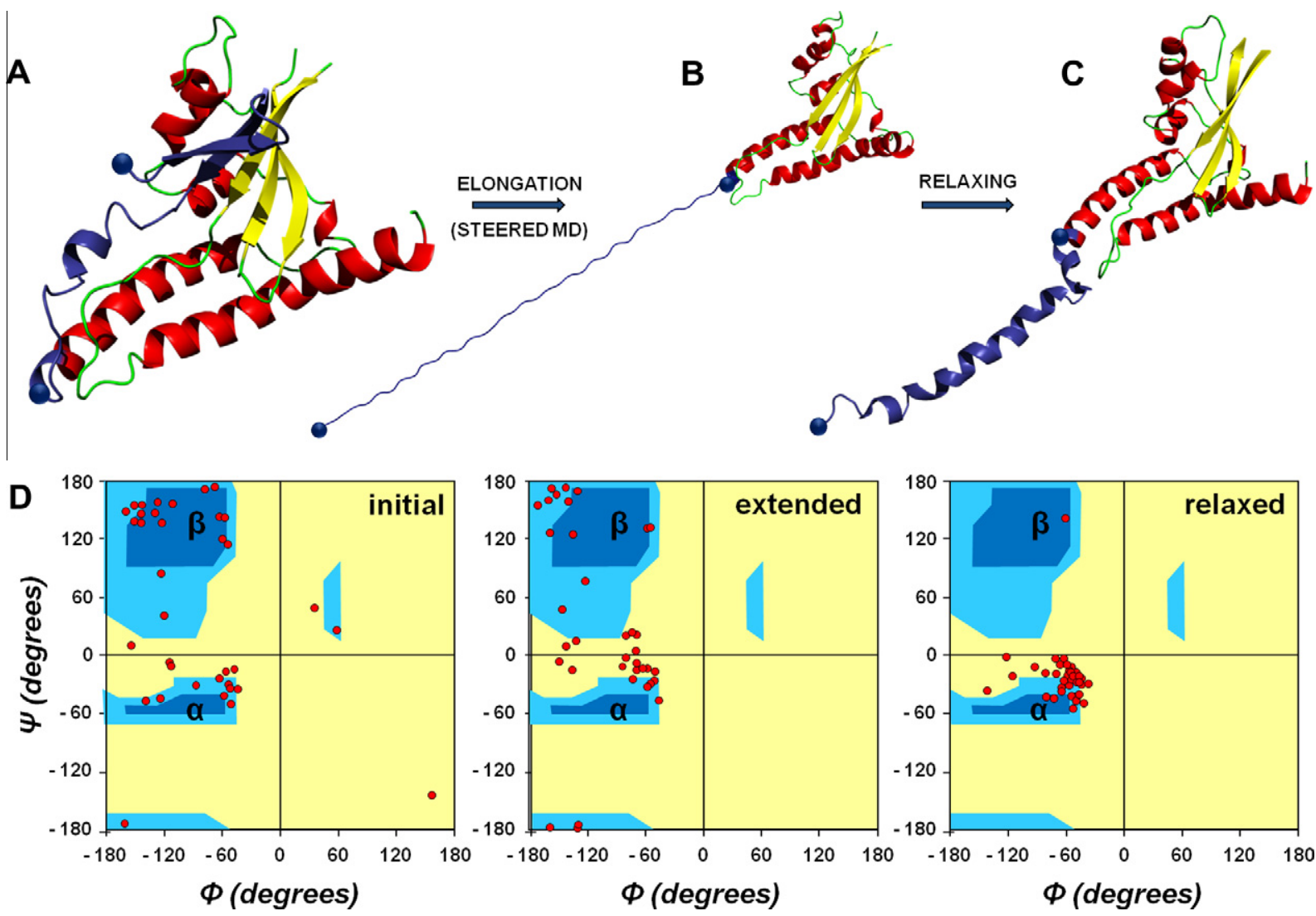

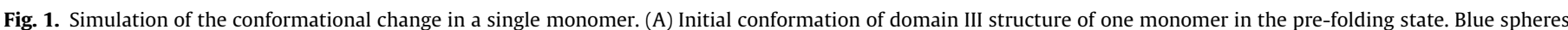

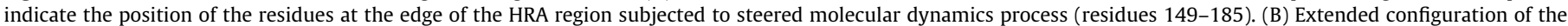

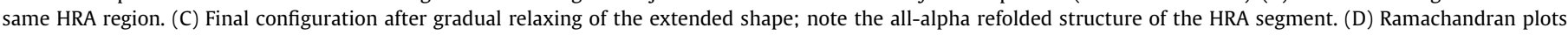

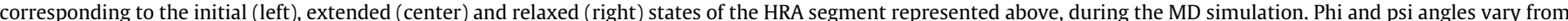

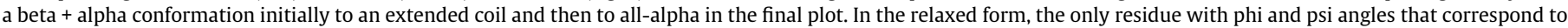
a beta conformation corresponds to Pro-178. (For interpretation of the references to color in this figure legend, the reader is referred to the web version of this article.)

the changes are mainly related to accommodating the newly formed $\alpha$-helix: they are less dramatic and do not involve changes in the secondary structure. In contrast to the well-known fusion event mechanism of orthomyxovirus [12], conformational change of paramyxovirus $\mathrm{F}$ protein is not dependent on $\mathrm{pH}$ [17] and unfortunately the published structures $[14,15]$ provide no suitable clue as to the mechanism behind the event that triggers fusion. In order to understand the pathway that the HRA region follows in passing from one structure to another, we ran a series of simulations using the pre-fusion state of the $\mathrm{F}$ protein from parainfluenza virus 5 (PDB: 2B9B [14]) as the starting structure, analyzing the steps that allow the HRA region to convert from one structure to another, and proposing a mechanism that drives the whole structural change based only on mechanical forces.

\section{Materials and methods}

\subsection{Protein structures}

Coordinates for the pre-fusion and post-fusion states of the trimeric form of $F$ protein were obtained from the Protein Bata Bank: PDB ID: 2B9B [14] and PDB ID: 1ZTM [15], respectively.

\subsection{Steered molecular dynamics}

Conformational change of $\mathrm{F}$ protein was simulated by steered MD procedures, using the SANDER module of the AMBER 10 package [18]. Generalized Born theory was applied to represent the solvent environment of the protein. All simulations were run taking Protein Data Bank entry 2B9B [14] as the starting structure. Disulfide bonds were assigned using the LEAP program. The steered MD process started with the adaptation of the initial structure to the AMBER force field through 5000 steps of energy minimization, using the conjugate gradient method combined with four initial steps of steepest descent in each iteration. This minimization was performed with no restrictions added to the initial structure. $\delta t$ was $0.001 \mathrm{ps}$. The system was then heated over 10,000 steps of equilibration, also with $\delta t=0.001 \mathrm{ps}$, which raised the temperature from 0 to $300 \mathrm{~K}$, while restraining the position of the C $\alpha$ atoms. Finally, torsion restrictions were removed from the segment spanning residues Ala-149 to Cys-185, in order to start the MD. Three kinds of guide parameters were used to perform the steered MD: distance, angle and torsion between the $\mathrm{C} \alpha$ atoms of the residues. The values of these parameters in the simulation of one single monomer were as follows: (1) distance between Ca149 and C $\alpha 185$, from 26 to $123 \AA$; (2) angle C $\alpha 149-C \alpha 185-C \alpha 47$, from $24^{\circ}$ to $156^{\circ}$; and (3) torsion values between axes formed by C $\alpha 149-C \alpha 185$ and $C \alpha 47-C \alpha 204$ from $28^{\circ}$ to $4^{\circ}$. In all cases, the initial values corresponded to those of the pre-fusion structure $2 \mathrm{~B} 9 \mathrm{~B}$ [14] and final values to those of the post-fusion structure 1ZTM [15]. Total simulation steps were $1.16 \times 10^{6}$. The force constant was $10 \mathrm{kcal} \mathrm{mol}^{-1} \AA^{-2}$. In this conditions, the energy added to the system in each computation step is lower than $2 \times 10^{-6} \mathrm{kcal} \mathrm{mol}^{-1}$ To simulate the whole trimer structure, the values were similar $\left(1.50 \times 10^{6}\right.$ steps $)$, only the respective angles for each monomer were varied to avoid atom collision during the elongation phase. 

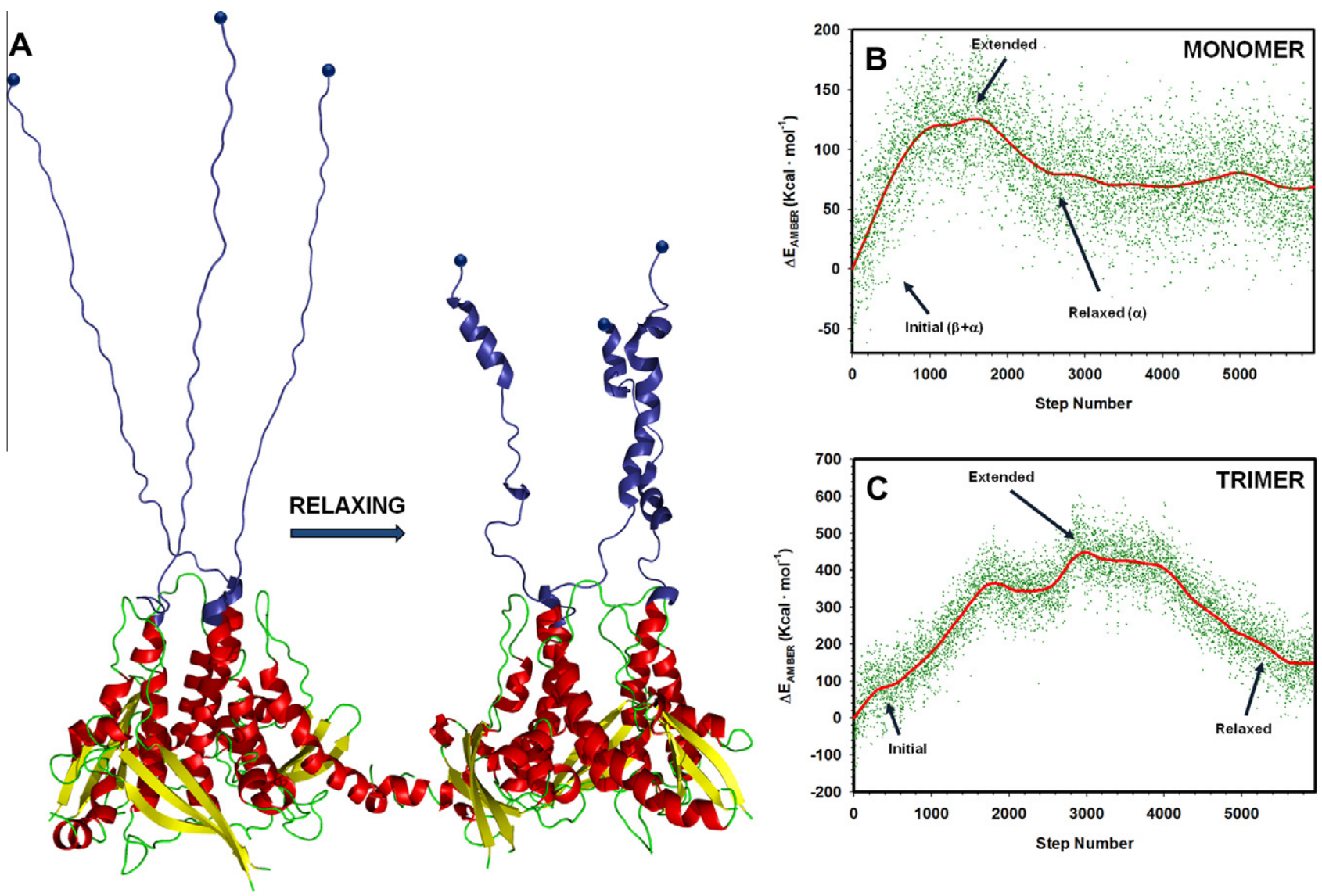

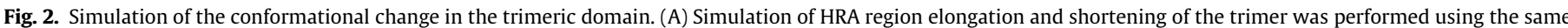

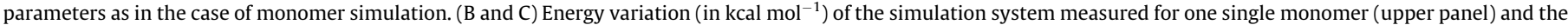
modeled trimer (lower panel). Approximate positions of the initial, extended and final relaxed states are indicated.

\subsection{Coiled coil formation}

To simulate the formation of the three-helix coiled coil, we performed a simple run of non-steered MD for $12 \mathrm{~ns}$, using a trimerized model of the single monomer structure resulting from the steered MD procedures as the initial structure. To simulate the interaction of the three fusion peptides in the cell membrane, a force of $10 \mathrm{kcal} \mathrm{mol}^{-1} \AA^{-2}$ was applied during the initial steps to the distal end of each chain as a distance restriction, to avoid opening of the three helices. Distance control was removed after the first $0.2 \mathrm{~ns}$ of the run to allow the $\alpha$-helices to interact without restrictions. Additional distance restrictions of the same value were applied to maintain the geometry between the C $\alpha 194, \mathrm{C} \alpha 55$ and C $\alpha 206$ atoms in the base of each monomer and thus to maintain the whole trimeric structure in the absence of the rest of the modeled protein.

\section{Results and discussion}

The behavior of the $\mathrm{F}$ protein trimer after the proteolytic event and subsequent binding of the three fusion peptides to the cell membrane was simulated using molecular dynamics (MD) techniques. In a first step, the individual change in the HRA region in DIII of one single monomer was simulated. Then, two alternative simulations were run taking into account the structure of DIII in the three monomers of the F protein, in order to analyze the formation of the coiled coil.

\subsection{Simulation of the conformational change in a single monomer}

In the first step, a simplified structure was used to explain the conformational change of HRA in a single monomer. Only DIII of
PDB structure 2B9B [14] was used as the starting construct: residues $42-279$ of $2 \mathrm{~B} 9 \mathrm{~B}$. A small fragment of HRA (residues 130-148, gray in Supp. Fig. 1), located immediately after the fusion peptide, was omitted from the MD simulation, as it does not undergo any conformational change during the protein fusion process and thus we were able to reduce the number of atoms in the system and reduce the computational cost of the subsequent simulation of the whole trimer (see below).

Unlike Orthomyxoviridae hemagglutinin protein, whose conformational change is caused by acidification in the endosome environment, no chemical variations, not even changes in $\mathrm{pH}$ [17], have been demonstrated to cause of the conformational change in paramyxovirus $\mathrm{F}$ protein. We hypothesized two possible triggering mechanisms. First, that the initial structure is synthesized in an unstable conformation that spontaneously changes to the pre-hairpin intermediate after the proteolytic process. In our hands, this hypothesis was initially ruled out as during unrestrained MD of DIII (and of the whole protein structure, see Supplementary Fig. S4) no substantial changes were observed, suggesting that the protein remains in a stable state, at least in the absence of external factors.

The second hypothesis is that an external force is the main cause of the structural change, driving the domain structure from the initial stable state to an unstable intermediate state (perhaps the extended intermediate state described previously [13]) and then to a subsequent stable state after the structural changes. As the fusion peptide was supposed to be included in the cell membrane, we hypothesized that, once attached, the random movement of the virus particles (in the target tissue or in the cell culture medium) would produce a putative stretching of the protein structure, and that this external mechanical force drives the structural rearrangement of DIII. 

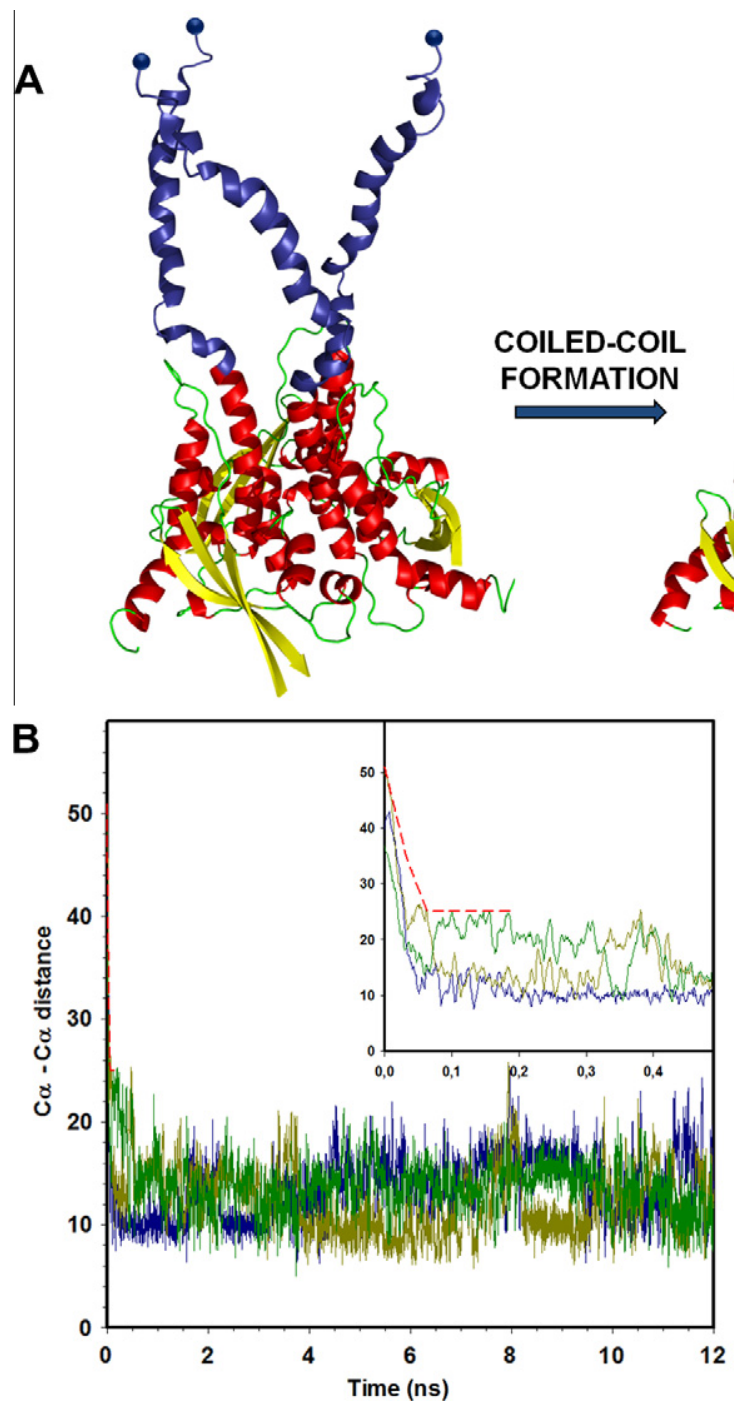
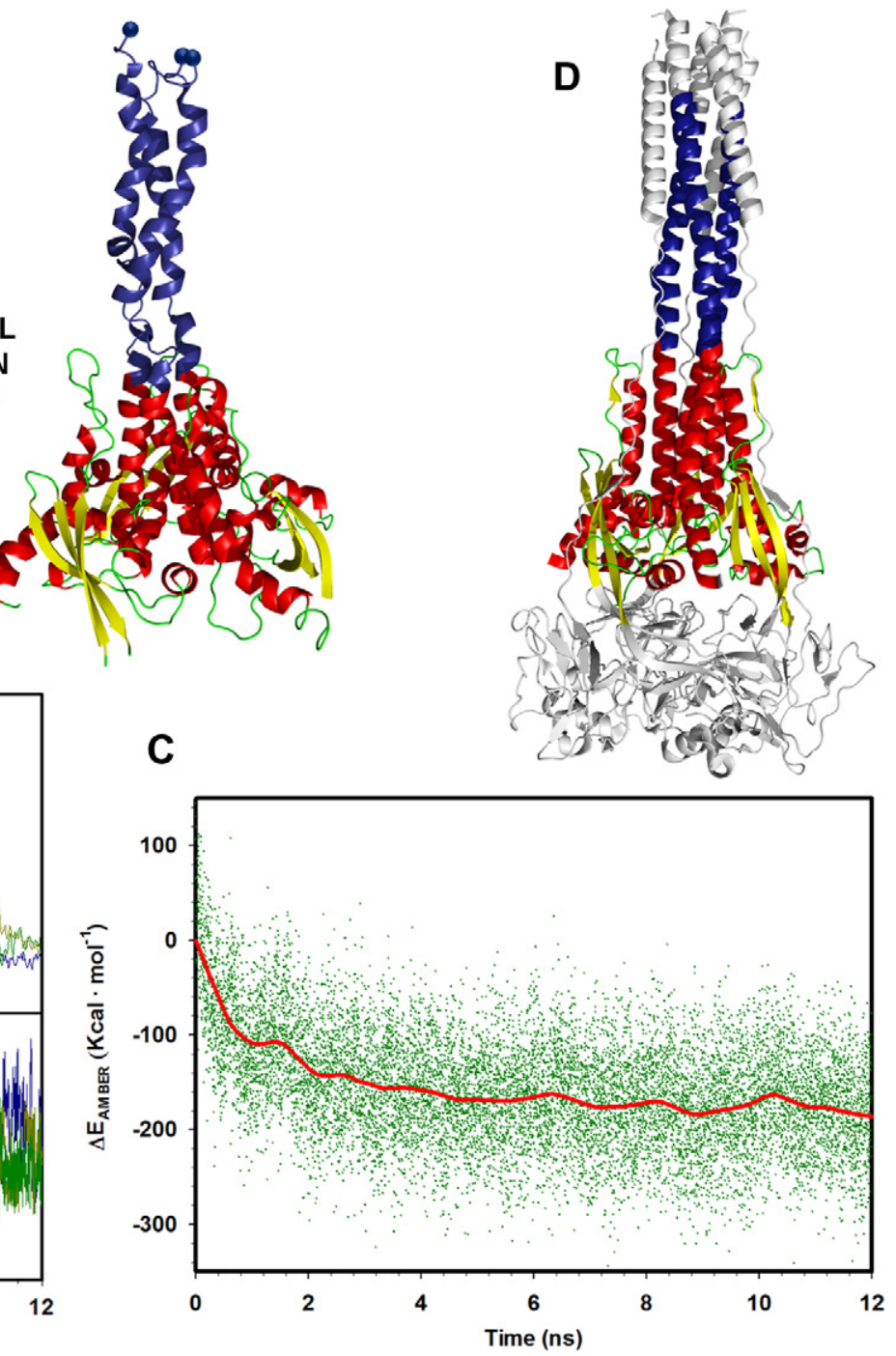

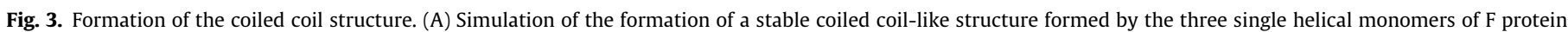

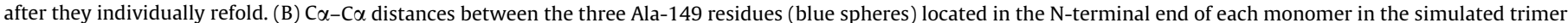

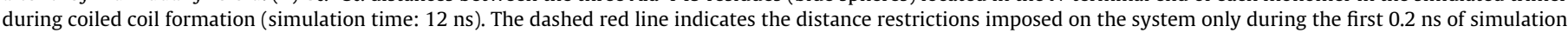

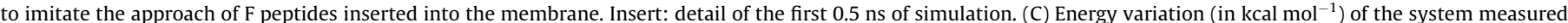

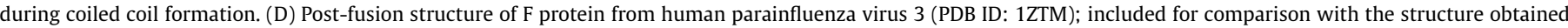

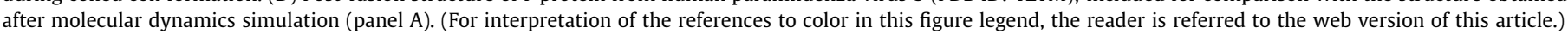

To study whether, under the conditions simulated, this latter hypothesis is plausible, the presence of a mechanical force was included in the simulation. Using steered MD, a force was applied to compel residues Ala-149 and Cys-185 to separate along their longitudinal axis (Fig. 1A and B, blue spheres). The mechanical force was only applied to these two residues because it is the region between them that experiences the greatest conformational change that differentiates the pre- and post-fusion structures (Supplementary Fig. S1, purple box). The MD parameters that steer the simulated conformational change are based on variations in distance, angle and torsion values between the $\mathrm{C} \alpha$ atoms of the Ala-47, Ala-149, Cys-185 and Thr-204 residues (see Section 2). The other residues that do not participate in the main structural change were maintained in their initial positions by imposing a restriction on the torsion values for their $\mathrm{C} \alpha$ atoms. The result of the elongation phase of the simulation, after increasing the distance between the $\mathrm{C} \alpha$ atoms of Ala-149 and Cys-185 from 26.05 to $135 \AA$, is shown in Fig. $1 \mathrm{~B}$; the initial structure of the HRA region ( 2 beta-strands plus $1 \alpha$-helix, PDB: 2B9B [14]) was reorganized into an extended coil. After the forced elongation phase, a subsequent step of slow relaxing (Fig. 1C) was simulated by reducing, stepwise, the distance between the C $\alpha$ atoms of Ala-149 and Cys185 from 135 to $64 \AA$. The most remarkable result from this phase was the spontaneous rearrangement of the extended coil into an all- $\alpha$-helix structure, as observed in the crystallized post-fusion structure (1ZTM [15]). Ramachandran plots generated for the initial, elongated and final states of the HRA segment (Fig. 1D) illustrate the reorganization of the segment, where the phi and psi angles change from those of a beta-strand to those of a coil and then to an $\alpha$-helix. These results indicate that, at least in one monomer, applying only a simulated mechanical force to the initial folded structure of the HRA region, this segment can completely change its beta + alpha configuration to an extended one and then, after relaxation, to an all-alpha structure, very similar to the actual post-fusion structure [15]. To analyze if the trajectory has reached an overall sufficient sampling, non-weighted covariance matrix of $\mathrm{C} \alpha$ atoms of the structure was calculated to obtain cosine content $\left(c_{\mathrm{i}}\right)$ [19] for the first principal components (Supplemental 


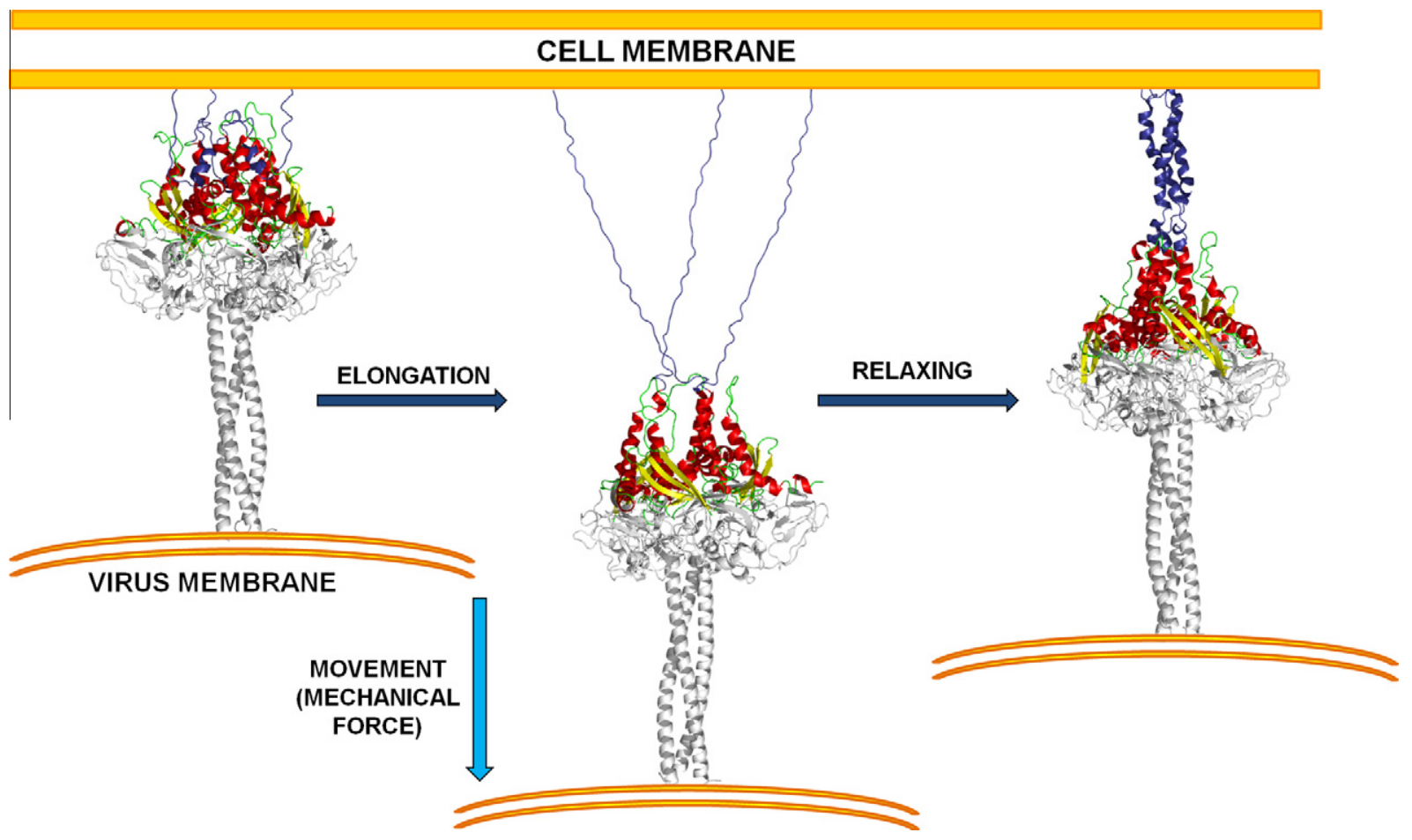

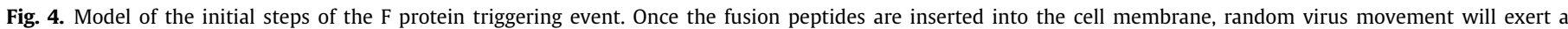

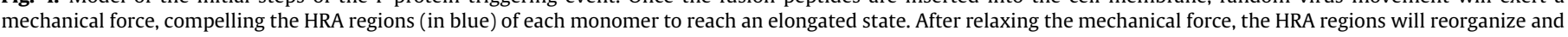

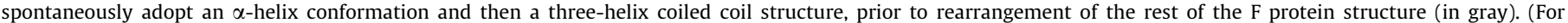
interpretation of the references to color in this figure legend, the reader is referred to the web version of this article.)

Table S1). This value ranges between 0 (no cosine) and 1 (perfect cosine). Values close to 1 are representative of random motion, and therefore, of insufficient sampling For our system, the $c_{\mathrm{i}}$ values indicated that the sampling of the performed conformational space is sufficient to observe a stable structural change. Detailed analysis of the steered-MD parameters is shown in Supplemental Fig. S2.

\subsection{Simulation of the conformational change in the trimeric domain}

Once the behavior of a single monomer had been analyzed, our next step was to analyze the performance of the native trimeric form of DIII. It is accepted, and it has previously been demonstrated for paramyxovirus using peptides that mimic regions HRA and HRB [20], that the formation of the pre-hairpin intermediate structure includes the generation of a three-helix coiled coil involving the three HRA regions before the formation of the definitive six-helix bundle, which is believed to appear only after the final membrane fusion event. In contrast, there is no evidence that indicates whether the formation of the three-HRA coiled coil occurs at the same time as or after the formation of each individual HRA $\alpha$-helical structure. To analyze these two options, two different simulations were performed for the re-folding of the three HRA regions after the initial stretching step. The first approach consisted of simultaneously elongating the three peptides, in the same manner as described previously for a single monomer, and then relaxing the three extended coils at the same time, allowing them to reach equilibrium. The results, summarized in Fig. 2A, indicated that, although the three coils became rearranged into structures with a high percentage of $\alpha$-helical segments, complete helical structures were not generated and that after the equilibrium at the final length was reached, there was no formation of the expected coiled coil.

Despite this, the MD procedure simulated the formation of $\alpha$ helices in several continuous segments of the HRA region of the three monomers. Measurement of the variation of total energy values for the monomeric structure throughout the whole simulated trajectory (Fig. 2B) suggested the presence of an energy barrier, with a maximum in the extended conformation between the two folded states of the HRA region (pre- and post-fusion). The value of the measured energy barrier was around $180 \mathrm{kcal} \mathrm{mol}^{-1}$. The values obtained during the MD simulation of the trimer (Fig. 2C) exhibit a very similar profile to those obtained in the simulation of the monomer. They are also self-consistent, as the values for the trimeric form are roughly threefold higher than those obtained for a single monomer. In agreement with this, the value of the energy barrier of the trimer was around $450 \mathrm{kcal} \mathrm{mol}^{-1}$.

\subsection{Coiled coil formation}

Although the results of the first approach indicated the possibility of the formation of helical segments in the HRA region, the generation of stable coiled coils was not observed during the simulation. The second approach consisted of generating a trimeric form of DIII using as the starting structure the final relaxed form obtained in the simulation of the single monomer. The modeled trimer (Fig. 3A, left) was then subjected to MD. To simulate the interaction of the three fusion peptides in the cell membrane, a distance restriction was applied to the distal final end of each helical peptide (Fig. 3A, blue spheres) only during the initial steps of the MD. Continuous measurement of the distances between the $\mathrm{C} \alpha$ atoms of the three residues (Fig. 3B) indicated that after the first nanosecond (distance boundaries are indicated by a red line in Fig. 3B), the values settled into an equilibrium that was maintained without restrictions for the rest of the simulation. As expected, the measurement of the variation of energy values during the simulation process showed a continuous reduction towards a more stable level (Fig. 3C). The structure resulting from the MD (Fig. 3A, right) is consistent with a trimeric HRA coiled coil and it is remarkably 
analogous (Fig. 3D) to the actual crystallized assembly of this segment in the structure of the post-fusion $F$ protein of paramyxoviruses [15]. This result, compared to that presented in Fig. 2, suggests that, at least using MD simulation procedures, the formation of the three individual $\alpha$-helices needs to be completed before the generation of the definitive coiled coil structure. Detailed analysis of the MD simulation parameters is shown in Supplemental Fig. S3. Also, as it was also measured for the monomer, the cosine content $\left(c_{\mathrm{i}}\right)$ [19] for the first principal components (Supplemental Table S1) indicated sufficient sampling of the conformational space.

In the present work, MD methods are used to devise a model of how conformational change of the HRA region of $F$ protein is produced during the first steps of the fusion of viral and cell membranes in paramyxoviruses. The model suggests that the cause of the reorganization could be a mechanical force that elongates the HRA segment, followed by relaxation and refolding of the region into an all-alpha state. The two stable conformations are separated by an energy barrier that, in addition to the stabilization of the final coiled-coil structure, ensures the irreversibility of the process. Fig. 4 summarizes the proposed model. In the initial steps, the fusion peptide is inserted into the cell membrane. Once anchored, the stretching of the HRA region is triggered by the movement of the virus (i.e. during diffusive or drifting motions on the plasma membrane [21]) driving the conformational change to an extended coil structure. A second movement of the virus allows the HRA region to relax and to refold into three helical structures, forming the characteristic three-helix coiled coil of the pre-hairpin structure [12].

\section{Acknowledgments}

We thank Dr. Galo Ramírez for his continued scientific advice and encouragement. We are grateful to Toffa Evans for valuable assistance in the preparation of the manuscript. We thank Dr. Elena Papaleo for her constructive help in the analysis of the MD trajectories. This study was supported by: the Spanish Ministerio de Ciencia e Innovación through Grants SAF2007-61926, IPT2011-0964900000 and SAF2011-13156-E; the Madrid regional authority through Grant S-BIO-0260/2006-COMBACT; and the European Commission through Grants FP7 HEALTH-F3-2009-223431 (EU Project "Divinocell") and FP7 HEALTH-2011-278603 (EU Project "Dorian"). Support from the "Fundación Ramón Areces" is acknowledged. We also thank the Centro de Computación Científica "CCCUAM" for computational support. Work at Biomol-Informatics was partially financed by the European Social Fund.

\section{Appendix A. Supplementary data}

Supplementary data associated with this article can be found, in the online version, at doi:10.1016/j.bbrc.2012.02.112.

\section{References}

[1] P.L. Collins, J.E. Crowe, Respiratory syncytial virus and meta-pneumovirus, in: D.M. Knipe, P.M. Howley, D.E. Griffin, R.A. Lamb, M.A. Martin, B. Roizman, S.E. Straus (Eds.), Fields Virology, Lippincott Williams and Wilkins, Philadelphia, PA, 2007. pp. 1601-1646.

[2] R.A. Lamb, T.S. Jardetzky, Structural basis of viral invasion: lessons from paramyxovirus F, Curr. Opin. Struct. Biol. 17 (2007) 427-436.

[3] R.A. Lamb, R.G. Paterson, T.S. Jardetzky, Paramyxovirus membrane fusion: lessons from the F and HN atomic structures, Virology 344 (2006) 30-37.

[4] C.K. Navaratnarajah, N. Oezguen, L. Rupp, L. Kay, V.H. Leonard, W. Braun, R. Cattaneo, The heads of the measles virus attachment protein move to transmit the fusion-triggering signal, Nat. Struct. Mol. Biol. 18 (2011) 128-134.

[5] R.K. Plemper, M.A. Brindley, R.M. Iorio, Structural and mechanistic studies of measles virus illuminate paramyxovirus entry, PLoS Pathog. 7 (2011) e1002058.

[6] K. Tanabayashi, R.W. Compans, Functional interaction of paramyxovirus glycoproteins: identification of a domain in Sendai virus HN which promotes cell fusion, J. Virol. 70 (1996) 6112-6118.

[7] L Gonzalez-Reyes, M.B. Ruiz-Arguello, B. Garcia-Barreno, L Calder, J.A Lopez J.P. Albar, J.J. Skehel, D.C. Wiley, J.A. Melero, Cleavage of the human respiratory syncytial virus fusion protein at two distinct sites is required for activation of membrane fusion, Proc. Natl. Acad. Sci. USA 98 (2001) 9859-9864.

[8] J. Rawling, O. Cano, D. Garcin, D. Kolakofsky, J.A. Melero, Recombinant Sendai viruses expressing fusion proteins with two furin cleavage sites mimic the syncytial and receptor-independent infection properties of respiratory syncytial virus, J. Virol. 85 (2011) 2771-2780.

[9] R.M. Schowalter, S.E. Smith, R.E. Dutch, Characterization of human metapneumovirus $\mathrm{F}$ protein-promoted membrane fusion: critical roles for proteolytic processing and low pH, J. Virol. 80 (2006) 10931-10941.

[10] G. Zimmer, L. Budz, G. Herrler, Proteolytic activation of respiratory syncytial virus fusion protein. Cleavage at two furin consensus sequences, J. Biol. Chem. 276 (2001) 31642-31650.

[11] M.L. Bissonnette, J.E. Donald, W.F. DeGrado, T.S. Jardetzky, R.A. Lamb, Functional analysis of the transmembrane domain in paramyxovirus $F$ protein-mediated membrane fusion, J. Mol. Biol. 386 (2009) 14-36.

[12] S.C. Harrison, Viral membrane fusion, Nat. Struct. Mol. Biol. 15 (2008) 690698.

[13] M. Porotto, C.C. Yokoyama, G. Orefice, H.S. Kim, M. Aljofan, B.A. Mungall, A. Moscona, Kinetic dependence of paramyxovirus entry inhibition, J. Virol. 83 (2009) 6947-6951.

[14] H.S. Yin, X. Wen, R.G. Paterson, R.A. Lamb, T.S. Jardetzky, Structure of the parainfluenza virus $5 \mathrm{~F}$ protein in its metastable, prefusion conformation, Nature 439 (2006) 38-44.

[15] H.S. Yin, R.G. Paterson, X. Wen, R.A. Lamb, T.S. Jardetzky, Structure of the uncleaved ectodomain of the paramyxovirus (hPIV3) fusion protein, Proc. Natl. Acad. Sci. USA 102 (2005) 9288-9293.

[16] X. Zhao, M. Singh, V.N. Malashkevich, P.S. Kim, Structural characterization of the human respiratory syncytial virus fusion protein core, Proc. Natl. Acad. Sci. USA 97 (2000) 14172-14177.

[17] S. Herfst, V. Mas, L.S. Ver, R.J. Wierda, A.D. Osterhaus, R.A. Fouchier, J.A. Melero, Low-pH-induced membrane fusion mediated by human metapneumovirus $\mathrm{F}$ protein is a rare, strain-dependent phenomenon, J. Virol. 82 (2008) 88918895.

[18] D.A. Case, T.E. Cheatham 3rd, T. Darden, H. Gohlke, R. Luo, K.M. Merz Jr., A Onufriev, C. Simmerling, B. Wang, R.J. Woods, The Amber biomolecular simulation programs, J. Comput. Chem. 26 (2005) 1668-1688.

[19] B. Hess, Convergence of sampling in protein simulations, Phys. Rev. E: Stat. Nonlin. Soft Matter Phys. 65 (2002) 031910.

[20] M. Magro, D. Andreu, P. Gomez-Puertas, J.A. Melero, C. Palomo, Neutralization of human respiratory syncytial virus infectivity by antibodies and lowmolecular-weight compounds targeted against the fusion glycoprotein, J. Virol. 84 (2010) 7970-7982.

[21] C.J. Burckhardt, U.F. Greber, Virus movements on the plasma membrane support infection and transmission between cells, PLoS Pathog. 5 (2009) e1000621. 